\title{
A NOVEL SCHEME FOR ACCURATE REMAINING USEFUl LIFE PREDICTION FOR INDUSTRIAL IOTS BY USING DEEP NEURAL NETWORK
}

\author{
Abdurrahman Pektaş ${ }^{1}$ and ElifNurdan Pektaş ${ }^{2}$ \\ ${ }^{1}$ Department of Computer Engineering, Galatasaray University, İstanbul, Turkey. \\ ${ }^{2}$ Siemens CT Turkey, Yakack Caddesi No: 111, 34870 Kartal, Istanbul, Turkey.
}

\begin{abstract}
In the era of the fourth industrial revolution, measuring and ensuring the reliability, efficiency and safety of the industrial systems and components are one of the uppermost key concern. In addition, predicting performance degradation or remaining useful life (RUL) of an equipment over time based on its historical sensor data enables companies to greatly reduce their maintenance cost. In this way, companies can prevent costly unexpected breakdown and become more profitable and competitive in the marketplace. This paper introduces a deep learning-based method by combining CNN(Convolutional Neural Networks) and LSTM (Long Short-Term Memory)neural networks to predict RUL for industrial equipment. The proposed method does not depend upon any degradation trend assumptions and it can learn complex temporal representative and distinguishing patterns in the sensor data. In order to evaluate the efficiency and effectiveness of the proposed method, we evaluated it on two different experiment: RUL estimation and predicting the status of the IoT devices in 2-week period. Experiments are conducted on a publicly available NASA's turbo fan-engine dataset. Based on the experiment results, the deep learning-based approach achieved high prediction accuracy. Moreover, the results show that the method outperforms standard well-accepted machine learning algorithms and accomplishes competitive performance when compared to the state-of-the art methods.
\end{abstract}

\section{KEYWORDS}

Industrial IoT, Remaining Useful Life, Predictive Maintenance, Deep Learning, Industry 4.0, Prognostics and Health Management

\section{INTRODUCTION}

Due to the increased competition and competitive pressures, industries try to explore new ways to maximize their profits while remain competitive in the marketplace. To this end, they primarily reduce their cost of production, especially their maintenance operations which is one of the most expenditure operation and cost about $15 \%-70 \%$ of their overall expenses [1]. This motivates companies to investigate more feasible maintenance strategies than standard maintenance, i.e. corrective and preventive. Because these two methods cannot suppress the unexpected breakdowns which results production lost, failed delivery scheduled and loss of reputation. For these reasons, companies adopting proactive maintenance approach called predictive maintenance. Predictive maintenance involves constantly monitoring the current state of the industrial equipment and predicting RUL of them. Predictive maintenance not only minimize failure risk but also maximize useful life of assets. In this context, accurately estimating RUL of an industrial equipment is one of the key factors for a company to become profitable and competitive.

Existing remaining useful life prediction methods have already made remarkable process in predictive maintenance. However, due to the complexity and diversity of industrial IoT devices, 
International Journal of Artificial Intelligence and Applications (IJAIA), Vol.9, No.5, September 2018

existing methods have some limitations. First and foremost, on the one hand, most of the existing methods are heavily depend on physical model or used complex signal processing methods, which involvein dept expert knowledge and labor. On the other hand, mostdata-driven methodsespecially which rely on standard machine learning algorithms have insufficient RUL detection capability because of the large volume of feature set and sensor data generated by IoT devices. To address these limitations, in this paper we proposed a deep learning-based RUL prediction and classification approach. Recently, deep neural network has become one of the most appealing methods in modeling and solving complex problems including such speech $[2,3]$ and image processing [4], video segmentation [5], etc. The proposed methodcombines CNN and LSTM neural networks to predict RUL trend for industrial equipment and does not depend upon any degradation trend assumptions. The method can learn complex temporal representative and distinguishing patterns in the sensor data.The study is also compared our with existing studies which employ the same dataset. According to the experimental results, the proposed deep architecture method achieved competitive RUL estimation performance.

The remaining of the paper is organized as follows: Section 2 presents related work on the subject of industrial IoT prognostic approaches. TheSection 3 introduces the proposed approach to estimate the remaining useful life of the industrial IOT device and the predicting of the state of IoT device in last two weeks period.In Section 4, we evaluate in detail the presented approach including the pre-processing procedure, description of the dataset and the result of the experiments.Section 4 also compares the standard machine learning methods with our deep learning-based solution. Finally, we draw some conclusions and point out possible directionsfor future research in Section 5.

\section{RELATED WORK}

The estimation of the RUL is broadly categorized into two groups physics-driven and data-driven [6]. Physics-driven approaches characterize degradation progress of an equipment through building mathematical models based on its failure mechanisms. Physics-driven approaches associate with the component properties and stress levels [7, 8, 9]. Whereas, data-driven approaches focus on learning degradation patterns from historical observations by using machine learning techniques. Currently, the data-driven models attack more attention than physics-based approaches from researchers because on the one hand they are very feasible and capable of modelling the dynamics of degradation process from sufficient historical observation. On the other hand, the physics-driven approaches require the domain knowledge about the root cause of all degradation processes $[10,11]$.

In the literature, various data-driven approaches such as support vector machines (SVMs) [12], Kalman filters (KF) $[13,14]$ and neural network $[15,16]$ has been widely used by researchers. However automatically estimating RUL based on sensor data such as temperature, vibration, etc., is a challenging task because of changing dynamic conditions. For example, daily of seasonal picks in the usage of a machine may lead to misleading predictions. To overcome this problem, deep learning can be used. Deep learning is an emerging field of study and may offer the best solution for many problems in various domains such as image and natural language processing, speech recognition [17]. One of the most important properties of deep learning is that once a neural network is trained with a massive amount of data, the ANN generates more accurate prediction results. Another key advantage of the deep learning is that although the feature engineering is the fundamental step of traditional machine learning methods, knowledge about particular domain is not required to select the feature set. In consequence, we leverage deep learning such that better accuracy rate can be obtained for RUL estimation. 
[18] introduces a feature selection method to cope with curse of dimensionality problem $[19,20]$. More specifically, the authors seek minimum feature set which preserves domain knowledge while improving the prediction performance and reducing runtime overhead. The proposed method applied agglomerative hierarchical clustering on feature set to group based on high correlation. Following, entropy based logistic distribution is performed on each correlated group. In each group, features which exceeds certain threshold are selected as discriminative feature.

Currently, the deep learning methods have been one of the primary focus of the researcher for estimation RUL. In [21], authors proposed multi-objective deep neural network ensemble method to predict RUL for industrial machines. The presented method allows simultaneous evaluation of deep belief neural network respect to two objectives: accuracy and diversity. Babu et al. [22] introduce CNN based approach to predict RUL. Convolution is applied on temporal dimensional sensor data for the aim of learning higher-level of representation from raw sensor data. To this end, authors use a sliding window technique to create 2-dimensional representation from sensor signals. More recently, [23] combines gradient boosted trees and feed-forward NNs to estimate RUL by using raw sensor data. The proposed method has ability to extract features importance. In the study, the authors also evaluate and compare the performance different machine learning method including multi-layer perceptron, support vector regression and CNN model. In [24], LSTM networks - capable of understanding sequential patterns - is investigated to increase RUL prediction accuracy.

\section{METHOD}

The proposed method includes three main phases as summarized in Figure-1. Firstly, prior to training deep neural network, each type of sensor data is normalized by individually scaling between 0 and 1. To this end we use MinMaxScaler method provided by Scikit-learn library [25]. By this way, we prevent some feature from dominating the others and ensure each feature to equally contribute to the final prediction model. In this step, additionally, sequence of 30 consecutive cycle values of the sensor values for each engine are extracted by using n-gram approach. This representation is required because CNN needs at least two-dimensional array to process.

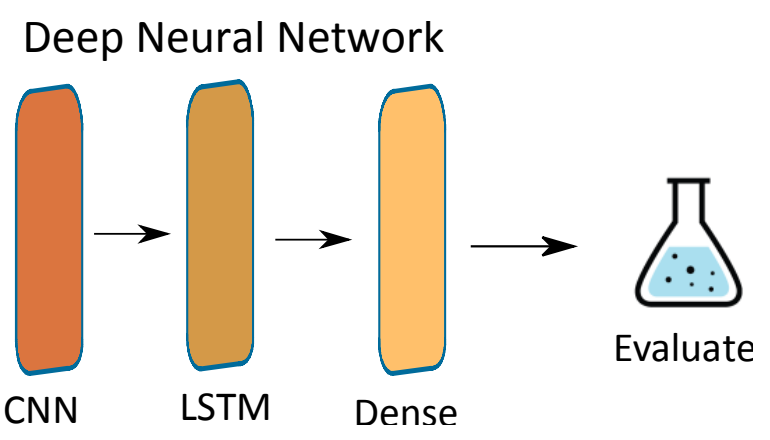

Figure 1. Overview of the proposed methodology to estimate RUL for industrial IoT devices.

In the second phase, the deep neural network is constructed based on the training dataset (broad overview of the architecture is depicted as dashed rectangle in Figure1). The convolutional part includes one dimensional convolution and a max pooling layer. On the one hand, the convolutional part derives hidden features from sensor data. On the other hand, convolution filter block extracts the relationship between input vectors and generates original features. We use convolution filter of size $3 \times 128$. After the convolutional layer, max-pooling is used to reduce the dimensionality of data. Outputs of the max-pooling layer are forwarded as an input to the LSTM layer, which is a particular type of the recurrent neural network (RNN) architecture. Then, LSTM 
layers are applied with 100 units to capture the sequential constraints in the sensor data. Then, the output of the LSTM layer is connected to the fully connected layer called dense layer. We use a dense layer consisting of 128 units. Finally, the dense layer predicts the RUL values and the state of the IoT stateby utilizing linear activation function for two experiment scenarios.

\section{EXPERIMENT AND RESULTS}

In this section, we present experimental results and compare with different machine learning approaches and some of the existing deep learning approaches. First, thedescribe of the evaluation dataset. Then, we define the evaluation metrics and compare the accuracy of the well-studied supervised algorithms such as SVM, random forest, etc. and the proposed approach. We evaluate the proposed approach on two different scenarios: RUL estimation and prediction of the status of a device in a 2-week period. Finally, the responsiveness of the proposed method is examined by comparing with some existing deep learning-based approaches.

\subsection{Dataset}

The proposed deep learning-based approach for RUL estimation is evaluated on publicly available Turbofan Engine dataset [26]. The benchmark dataset includes run-to-failure data for the multiple aero turbofan engines. Overall, there are 24 sensor data: 3 of them measure the external conditions such as fan stall margin and total temperature of high-pressure turbine (HPT) and remaining sensors measure internal conditions such as physical fan speed, engine pressure ratio, physical core speed, etc. The sensor values are composed of different types of features and units, such as the temperature (in ${ }^{\circ} \mathrm{R}$ ), pressure (in psia), speed (in psia) and bleed (in pps/psi).

The dataset is simulated and acquired from a virtual environment by using a software instead of real aero turbofan engines. Each training dataset includes 100 different engine and each engine has at least 100 observations. The dataset includes the whole time-varying operational conditions until the engine become unfunctional.

\subsection{Metrics}

To evaluate the effectiveness of the method and compare with some existing works, we used Mean Absolute Error (MAE) and Root Mean Square Error (RMSE) for RUL prediction. MAE scores the average magnitude of the error in RUL prediction. RMSE is a quadratic scoring method which gives equal penalize score to both early and late RUL estimation MAE. MAE and RMSE are defined as follows:

$$
\begin{array}{r}
M A E=\frac{1}{N} \sum_{i=1}^{N}\left|h_{i}\right| \\
R M S E=\sqrt{\frac{1}{N} \sum_{i=1}^{N} h_{i}^{2}}
\end{array}
$$

where $N$ is the number of engines and $h$ is the gap between the predicted RUL by the regression algorithm and the actual RUL value, $h=R U L_{\text {pred }}-R U L_{\text {actual }}$.

To evaluate second experiment which aims to detect the devices which fails in 2-week period. This task is a binary classification problem. The classifier predicts the status of the device as either functioning or failed in 2-week interval. F-measure, precision and recall metrics are used for this task. Recall (a.k.a positive predictive value) is the probability for a sample in class c to be 
classified correctly, the maximum value 1 means that the classifier is always correct about its prediction whether an instance belongs to class c. Precision gives the probability for an estimatedinstance about classification in class $\mathrm{c}$ to be actually in class c. Low precision means that a large number of samples are incorrectly classified in class c. In our case, a class can be labelled either as "functioning" or "failed".

The metrics are defined as follows:

$$
\begin{array}{r}
\text { precision }=\frac{t p}{t p+f p} \\
\text { Rrecall }=\frac{t p}{t p+f n} \\
F-\text { measure }=2 \frac{\text { precision } \cdot \text { recall }}{\text { precision }+ \text { recall }}
\end{array}
$$

where true positives (tp) refer to the correctly classified failed IoT devices while true negatives (tn) are the number of correctly classified functioning IoT devices in 2-week period. False positives (fp) refer the number of devicesincorrectly classified as failed devices which is actually in functioning state. Similarly, false negatives (fn) denote the number of functioning devices that are incorrectly classified as failed. In essence, the term positive and negative indicates the classifier's success whereas the term true and false denotes whether that particular prediction is matched with ground truth label or not.

\subsection{Results}

We use Keras [27] on top of Tensorflow [28] to implement the neural network architecture. We deploy the neural network on GPU version of Tensorflow which drastically reduces training time cost. All experiments are conducted on a workstation having Intel Xeon E5 $2600 \mathrm{v} 4$ processor, 64 GB DDR4 2133M HZ memory and two GPUs, namely GeForce 960 and the NVIDIA TITAN X GPU. To implement standard machine learning algorithms, Python Scikit learn library is used.

In the experiment, two different tasks are evaluated. In the first task, we predict the RUL for industrial devices. In the second task, we try to identify the running state of a given device in 2 weeks period, either functioning or failed. To this end, extensive experiments are conducted with various deep learning architectures in order to increase prediction accuracy. By combining a CNN, LSTM and fully connected layers, for the first task, we are able to obtain the best RUL prediction results with MAE of 10.2 and RMSE of 14.3 as shown in Table 1. According to the Table-1, the proposed deep learning method produces 3 times better performance. Also, we compare our results with some existing deep learning-based studies that also used C-MAPSS datasets for performance evaluation. As shown in Table 2, our system outperforms three of the four studies in terms of MAE and RMSE value and it also shows close performance metrics with [29]. We believe that the initial results can be further improved by tuning deep learning settings.

Table 1.Comparison of the performance of standard ML approaches

\begin{tabular}{|l|c|c|}
\cline { 2 - 3 } \multicolumn{1}{c|}{} & \multicolumn{2}{c|}{ Regression Task } \\
\hline Method & MAE & RMSE \\
\hline SVM & 39.57 & 53.12 \\
\hline RF & 30.21 & 42.18 \\
\hline K-NN & 31.00 & 43.36 \\
\hline The proposed method & 10.20 & 14.30 \\
\hline
\end{tabular}


Table 2.Comparison of the performance of some existing approaches which is evaluated onTurbofan Engine Data. NR: Not Reported.

\begin{tabular}{|l|l|l|l|}
\hline Study\& Year & Method & MAE & RMSE \\
\hline ESN-KF [30], 2012 & Echo state network & NR & 63.5 \\
\hline DeepCNN [22], 2016 & Deep CNN based regression & NR & 19.4 \\
\hline SOM [31], 2016 & Deep Feed-forward NN & NR & 17.2 \\
\hline Embed-LR [29], 2017 & Deep NN based on LSTM & 9.8 & 12.4 \\
\hline The proposed method, 2018 & Combination of CNN and LSTM & 10.2 & 14.3 \\
\hline
\end{tabular}

Table- 3 shows the performance results of the second task which is a binary classification problem. Each classifiers' class-wise results are given in terms of precision, recall and f-score. Based on the experiment, the proposed method achieved promising result while detecting possible failure in two weeks. Among the standard ML algorithm, KNN classifier achieve better classification results. Although each algorithm has similar classification result while predicting running state, they are generally failed while predicting possible failure. However, the proposed method detects effectively, and accurately possible breakdownsachieves the highest f-measure about \%96. Since this task is not addressed by studies used to compare the first task and their implementation is not open-source, we cannot evaluate this task with existing deep learning method.

Table 3.Comparison of the class-wise performance of standard ML and the proposed method while detecting possible breakdown of anindustrial equipment in two weeks.

\begin{tabular}{|l|l|c|c|c|}
\cline { 2 - 5 } \multicolumn{1}{c|}{} & \multicolumn{4}{|c|}{ Classification Task } \\
\hline \multirow{2}{*}{ SVM } & \multicolumn{1}{|c|}{ Class } & Precision & Recall & F-score \\
\hline \multirow{2}{*}{ RF } & Functioning & 0.99 & 0.99 & 0.99 \\
\cline { 2 - 5 } & Failed & 0.82 & 0.89 & 0.85 \\
\hline \multirow{2}{*}{ K-NN } & Functioning & 0.99 & 0.99 & 0.99 \\
\cline { 2 - 5 } & Failed & 0.82 & 0.86 & 0.84 \\
\hline \multirow{2}{*}{ The proposed method } & Functioning & 0.99 & 0.99 & 0.99 \\
\cline { 2 - 5 } & Failed & 0.83 & 0.89 & 0.86 \\
\cline { 2 - 5 } & Functioning & 0.99 & 0.99 & 0.99 \\
\cline { 2 - 5 } & Failed & 0.92 & 0.96 & 0.94 \\
\hline
\end{tabular}

\section{CONCLUSION AND FUtURE WORKS}

In this paper, we have proposed a deep learning approach for RUL estimation and classification. The proposed approach combines CNN and LSTM neural network to effectively discover hidden knowledge from raw sensor data. Experiments on NASA's jet engine degradation dataset (CMAPSS) demonstrate that the proposed method is effective and efficient in estimating RUL.When compared with respect to the state-of-the art studies and standard well-accepted machine learning approaches, the proposed method achieved competitive performance metric. Consequently, it can be concluded that the deep learning architecture can adequately learn latent degradation patterns.

In the future work, we are planning to apply the method on a real world industrial IoTs. Moreover, we will also concentrate on hyperparameter tuning for discovering the best performing deep learning setting. 


\section{ACKNOWLEDGEMENTS}

The authors are thankful to Galatasaray University for the facilities provided.The study is supported by Siemens Turkey. The authors also thank NASA Ames Research Center for sharing turbofan engine degradation dataset.

\section{REFERENCES}

[1] Bevilacqua, M., \&Braglia, M. (2000). The analytic hierarchy process applied to maintenance strategy selection. Reliability Engineering \& System Safety, 70(1), 71-83.

[2] Liu, W., Wang, Z., Liu, X., Zeng, N., Liu, Y., \&Alsaadi, F. E. (2017). A survey of deep neural network architectures and their applications. Neurocomputing, 234, 11-26.

[3] Schmidt, A., \& Wiegand, M. (2017). A survey on hate speech detection using natural language processing. In Proceedings of the Fifth International Workshop on Natural Language Processing for Social Media (pp. 1-10).

[4] Litjens, G., Kooi, T., Bejnordi, B. E., Setio, A. A. A., Ciompi, F., Ghafoorian, M., ... \& Sánchez, C. I. (2017). A survey on deep learning in medical image analysis. Medical image analysis, 42, 60-88.

[5] Garcia-Garcia, A., Orts-Escolano, S., Oprea, S., Villena-Martinez, V., Martinez-Gonzalez, P., \& Garcia-Rodriguez, J. (2018). A survey on deep learning techniques for image and video semantic segmentation. Applied Soft Computing, 70, 41-65.

[6] Lei, Y., Li, N., Guo, L., Li, N., Yan, T., \& Lin, J. (2018). Machinery health prognostics: A systematic review from data acquisition to RUL prediction. Mechanical Systems and Signal Processing, 104, 799-834.

[7] Cubillo, A., Perinpanayagam, S., \&Esperon-Miguez, M. (2016). A review of physics-based models in prognostics: Application to gears and bearings of rotating machinery. Advances in Mechanical Engineering, 8(8), 1687814016664660.

[8] Zhao, F., Tian, Z., \& Zeng, Y. (2013). Uncertainty quantification in gear remaining useful life prediction through an integrated prognostics method. IEEE Transactions on Reliability, 62(1), 146159.

[9] Wang, J., Gao, R. X., Yuan, Z., Fan, Z., \& Zhang, L. (2016). A joint particle filter and expectation maximization approach to machine condition prognosis. Journal of Intelligent Manufacturing, 1-17.

[10] Mosallam, A., Medjaher, K., \&Zerhouni, N. (2016). Data-driven prognostic method based on Bayesian approaches for direct remaining useful life prediction. Journal of Intelligent Manufacturing, 27(5), 1037-1048.

[11] Kan, M. S., Tan, A. C., \& Mathew, J. (2015). A review on prognostic techniques for non-stationary and non-linear rotating systems. Mechanical Systems and Signal Processing, 62, 1-20.

[12] Huang, H. Z., Wang, H. K., Li, Y. F., Zhang, L., \& Liu, Z. (2015). Support vector machine based estimation of remaining useful life: current research status and future trends. Journal of Mechanical Science and Technology, 29(1), 151-163.

[13] Miao, Q., Xie, L., Cui, H., Liang, W., \&Pecht, M. (2013). Remaining useful life prediction of lithiumion battery with unscented particle filter technique. Microelectronics Reliability, 53(6), 805-810. 
International Journal of Artificial Intelligence and Applications (IJAIA), Vol.9, No.5, September 2018

[14] Sikorska, J. Z., Hodkiewicz, M., \& Ma, L. (2011). Prognostic modelling options for remaining useful life estimation by industry. Mechanical Systems and Signal Processing, 25(5), 1803-1836.

[15] Tian, Z. (2012). An artificial neural network method for remaining useful life prediction of equipment subject to condition monitoring. Journal of Intelligent Manufacturing, 23(2), 227-237.

[16] Mahamad, A. K., Saon, S., \&Hiyama, T. (2010). Predicting remaining useful life of rotating machinery based artificial neural network. Computers \& Mathematics with Applications, 60(4), 1078-1087.

[17] Deng, L., \& Yu, D. (2014). Deep learning: methods and applications. Foundations and Trends ${ }^{\circledR}$ in Signal Processing, 7(3-4), 197-387.

[18] Aremu, O. O., Cody, R. A., \&McAree, P. R. Application of RelativeEntropy in FeatureSelectionforPredictiveMaintenance.

URL:

https://www.researchgate.net/publication/325615558_Application_of_Relative_Entropy_in_Feature_ Selection_for_Predictive_Maintenance.

[19] James, G., Witten, D., Hastie, T., \&Tibshirani, R. (2013). An introduction to statistical learning (Vol. 112). New York: springer.

[20] Pektaş, A., \&Acarman, T. (2017). Classification of malware families based on runtime behaviors. Journal of Information Security and Applications, 37, 91-100.

[21] Zhang, C., Lim, P., Qin, A. K., \& Tan, K. C. (2017). Multiobjective deep belief networks ensemble for remaining useful life estimation in prognostics. IEEE transactions on neural networks and learning systems, 28(10), 2306-2318.

[22] Babu, G. S., Zhao, P., \& Li, X. L. (2016, April). Deep convolutional neural network based regression approach for estimation of remaining useful life. In International conference on database systems for advanced applications (pp. 214-228). Springer, Cham.

[23] Singh, S. K., Kumar, S., \& Dwivedi, J. P. (2017). A novel soft computing method for engine RUL prediction. Multimedia Tools and Applications, 1-23.

[24] Wu, Y., Yuan, M., Dong, S., Lin, L., \& Liu, Y. (2018). Remaining useful life estimation of engineered systems using vanilla LSTM neural networks. Neurocomputing, 275, 167-179.

[25] Pedregosa, F., Varoquaux, G., Gramfort, A., Michel, V., Thirion, B., Grisel, O., ... \&Vanderplas, J. (2011). Scikit-learn: Machine learning in Python. Journal of machine learning research, 12(Oct), 2825-2830.

[26] Saxena, A., \& Goebel, K. (2008). Turbofan engine degradation simulation data set. NASA Ames Prognostics Data Repository.

[27] Chollet, F. (2015). Keras: Deep learning library for theano and tensorflow. URL: https://keras.io/.

[28] Abadi, M., Barham, P., Chen, J., Chen, Z., Davis, A., Dean, J., ... \&Kudlur, M. (2016, November). Tensorflow: a system for large-scale machine learning. In OSDI (Vol. 16, pp. 265-283).

[29] Gugulothu, N., TV, V., Malhotra, P., Vig, L., Agarwal, P., \& Shroff, G. (2017). Predicting Remaining Useful Life using Time Series Embeddings based on Recurrent Neural Networks. arXiv preprint arXiv:1709.01073.

[30] Peng, Y., Wang, H., Wang, J., Liu, D., \& Peng, X. (2012, June). A modified echo state network based remaining useful life estimation approach. In Prognostics and Health Management (PHM), 2012 IEEE Conference on (pp. 1-7). IEEE. 
[31] Macmann, O. B., Seitz, T. M., Behbahani, A. R., \& Cohen, K. (2016). Performing diagnostics \& prognostics on simulated engine failures using neural networks. In 52nd AIAA/SAE/ASEE Joint Propulsion Conference (p. 4807).

\section{Authors}

Abdurrahman Pektaş received his B.Sc. and M Sc. at Galatasaray University and his $\mathrm{PhD}$ at the University of Joseph Fourier, all in computer engineering, in 2009, 2012 and 2015, respectively. He is a senior researcher at Galata saray University. His research interests are analysis, detection and classification of malicious software, machine learning And security analysis tool development.

Elif Nurdan Pektaş received his B.Sc. and M Sc. at Galatasaray University all in computer engineering, in 2010, and 2014, respectively. She is leading software developer at Siemens Turkey. Her research interests are developing IoT based applications, deep learning, cloud based application and automated testing.
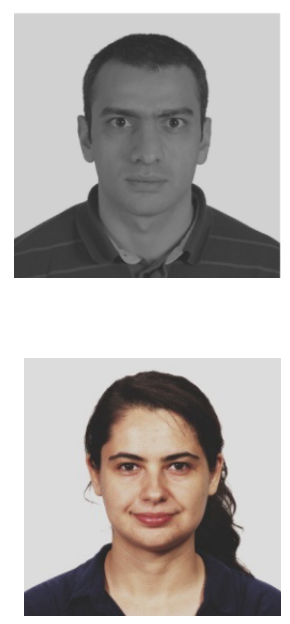